\section{RÉFÉRENCES}

1. Follea L. Une expertise officielle admet que l'amiante causera au moins 1950 décès en 1996. Le Monde, 3 juillet 1996, p. 16.

2. Follea L. L'étude menée par l'Académie de Médecine sur l'amiante lui vaut le dépôt d'une plainte. Le Monde, 14 novembre 1996, p. 11.

3. Jaurand M. Mésothéliome, amiante et cancer. Med Sci 1996 ; 12 : 1035-6.
4. Vaille C, Souchard M. Action hémolytique de l'amiante. Ann Pharm Fr 1978 ; 36 : 23-32.

5. Vaille C. La pollution de l'eau par l'amiante. Bulletin de l'Ordre des pharmaciens mars 1978, $\mathrm{n}^{\circ} 209$.

6. Vaille C. L'amiante et les installations classées. Anna. des Mines, juillet-août 1979, p. 47-60.

7. Vaille C. Amiante et cancer. Revue Française des Affaires Sociales, octobre décembre 1982 : $133-54$.

\section{Charles Vaille}

Inspecteur général honoraire de la santé, 233, rue de Vaugirard, 75015 Paris, France.

\section{COURRIER}

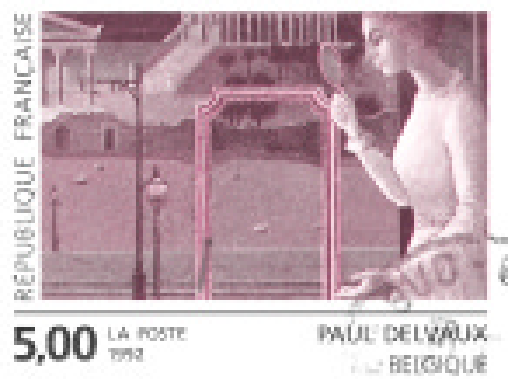

\title{
Application des principes de la toxicologie aux faibles niveaux d'amiantes
}

Le médecin toxicologue doit respecter les principes fondamentaux de la toxicologie et souhaite que les épidémiologistes fassent de même lorsqu'ils écrivent sur des maladies d'origine toxique.

Le premier principe en physiologie et en toxicologie (disciplines appliquées aux êtres vivants) est l'existence d'une dose-seuil reconnue pour obtenir un effet toxique, quel que soit le toxique, quel que soit l'effet à évaluer, quelle que soit l'association toxique ou nocive étudiée. Il n'existe aucune observation médicale, aucune étude expérimentale sur organisme vivant, bactéries comprises, qui contredise cette première notion.

Les épidémiologistes œuvrant en lement aider les médecins toxicologues à préciser la valeur de ce seuil pour chaque produit et chaque effet (cible).

Les deux cibles sont, pour les amiantes, le mésothéliome pleural et, problème tout différent, le cancer du poumon. Le mésothéliome n'est pas corrélé au tabagisme mais à l'accumulation de fibres dans la plèvre. En revanche, le facteur tabagisme est toujours dominant dans le cancer du poumon.

Le deuxième principe est la nécessité d'une identification exacte des substances étudiées (l'oxyde arsénieux n'est pas l'arsenic métal, la poussière de quartz n'est pas la poussière de silice amorphe). Depuis 20 ans la nocivité des amphiboles (fibres "pointues des deux bouts») est reconnue être bien supérieure à celle du chrysotile (sinueux).

Le troisième principe est l'identification ou l'évaluation aussi précise que possible de la dose absorbée («la dose fait le poison ») et, d'abord, des doses inhalées: c'est très facile en France pour des groupes bien surveillés par les médecins du travail, pour lesquels les niveaux d'intoxication sont mesurés depuis 20 ans, facilement évalués depuis 40 ans.

1 fibre $/$ litre $=2$ parties par million = inhalation de $1,6 \mathrm{mg}$ d'amiante en 100 ans: inhalation ne signifie pas absorption

Les niveaux d'empoussiérage ayant provoqué des cancers expérimentaux chez l'animal sont de l'ordre de 
$10 \mathrm{mg} / \mathrm{m}^{3}$, soit 5 millions de fibres par litre, ce qui est un taux trop élevé pour autoriser une transposition de l'animal à l'homme.

Ma divergence avec le rapport Inserm est fondamentale et concerne les modèles utilisés par les épidémiologistes. Sans se référer aux notions acquises en toxicologie, les épidémiologistes Inserm utilisent un modèle dit d'extrapolation linéaire à l'origine. C'est à la fois une contrevérité et un sophisme car les conclusions pratiques qui en sont tirées conduisent à des comportements qui présentent l'inconvénient de faire apparaître des dangers là où il n'y en a pas (pas de risque nul) puis à imposer des précautions là ou c'est inutile (précaution illimitée d'où responsabilité illimitée).

$\mathrm{Si}$, aux biologistes moléculaires que sont de nombreux lecteurs de médecine/sciences, je disais que, par extrapolation, un Becquerel est dangereux ou bien ils fermeraient leurs laboratoires, car tous les laboratoires devraient alors être fermés en raison de leur dangerosité potentielle, ou bien ils me riraient au nez en me disant que ma proposition est absurde.

Les épidémiologistes doivent s'aider de modèles à la condition que ceuxci concordent avec le maximum de données scientifiques et médicales. Les modèles avec seuil sont les seuls représentant la réalité physiologique de la toxicologie de l'être vivant.

\section{Les modèles sans seuil doivent être rejetés}

La méthode de calcul de risque toxicologique par extrapolation linéaire à l'origine s'écarte du graphe des observations réelles dès la plus petite dose et impose deux raisonnements d'abord faux puis pervers s'ils sont manipulés sans le moindre esprit critique. Selon cette méthode, il n'existerait pas de dose à effet nul, donc jamais de risque nul. Voyons ce que donnerait cette méthode appliquée à une maladie professionnelle bien connue, la silicose des sableurs. S'il n'y a pas de risque nul quelle que soit l'exposition, la plage de sable devient obligatoirement cause de silicose chez les enfants et le risque peut être calculé: par exemple, on conclut que dix enfants doivent être atteints de silicose tous les ans. On touche là le caractère singulier de la méthode retenue dans le rapport de l'Inserm. L'inconvénient sociologique majeur de l'extrapolation linéaire à zéro (ELZ), outre sa contradiction avec les lois fondamentales de la régulation de tous les êtres vivants qui n'intéresse que les scientifiques, est "l'interdiction totalitaire" du risque nul et, inversement, "l'introduction totalitaire» d'une lutte illimitée contre toutes les substances jugées étrangères (lutte inutile pour protéger la santé, désastreuse pour l'économie d'un pays, et poursuivie sans trève car elle est auto-entretenue, alimentée par - et alimentant - la peur chimique).

\section{Extrapolations en cascade}

Par l'extrapolation à zéro, nous avons vu que la représentation d'un risk, traduit avec le contresens «risque" en français, peut être rattaché à la plus petite unité, ce qui signifie que le lecteur de bonne foi croira qu'une fibre d'amiante par litre d'air peut entraîner, effectivement, l'apparition de $n$ mésothéliomes. En multipliant ce taux unitaire imaginaire par le nombre de fibres et par le nombre d'habitants, on obtient un nombre de cancers, toujours aussi imaginaires, développés dans la population (570, par exemple), mais en les donnant comme bien réels puisqu'il s'agit de centaines pour les médias. D'où ma demande de s'en tenir à la toxicologie quand il s'agit de textes toxicologiques. Car je rappelle qu'il n'existe aucune observation de maladie cancéreuse due à l'amiante - en particulier due au chrysotile - pour un taux nettement inférieur à
1000 fibres par litre d'air, a fortiori pour un taux inférieur à 100 fibres/ litre d'air (exposition 8 heures par jour).

Les informations et recommandations devraient donc, selon moi, être très rassurantes sauf dans le cas exceptionnel de dépassement de ces taux. C'est dans ce cas qu'il faut décider de modifications profondes, éventuellement de la suppression du bâtiment, ce que l'Académie nationale de médecine a clairement indiqué dans sa recommandation pratique de 1996 qui oriente surtout vers la surveillance et l'entretien réguliers des bâtiments amiantés, vers des améliorations limitées de mise en conformité, en s'efforçant de garantir en même temps la sécurité amiante des personnels du site et du voisinage et la sécurité incendie, souvent autrement préoccupante que la sécurité amiante. Le principe de précaution, très prisé actuellement, n'a aucun sens scientifique. Si le danger n'existe pas, aucune précaution n'est nécessaire. Il s'agit de simple phraséologie politique.

Selon les règles de l'éthique et de la déontologie médicale (et même du droit commun), il revient aux scientifiques de refuser de proposer aux médias des informations contestables, terrifiantes et simplistes, relevant de la science-fiction

\section{Rapports cités ou rappelés.}

Expertise collective Inserm (Bureau de presse et de relations publiques), Paris 1996 (bibliographie à demander). Rapport amiante, Bull Acad Nat Med Paris (Académie nationale de médecine), Paris 1996 (sans bibliographie), le rapport étant rédigé comme tous les rapports ANM par des personnes disposant déjà de leur bibliographie.

\section{P. Étienne Fournier}

Professeur honoraire de clinique toxicologique MANM, hôpital Fernand-Widal. 200, rue du Faubourg-Saint-Denis, 75010 Paris, France. 